(1)

CrossMark

\title{
Hot off the press: downregulation of PRMT1 for long-lasting effects of bronchial thermoplasty
}

\author{
Siti Farah Rahmawati ${ }^{1,2}$ and Reinoud Gosens ${ }^{1}$ \\ Affiliations: ${ }^{1}$ Dept of Molecular Pharmacology and GRIAC Research Institute, University of Groningen, \\ Groningen, The Netherlands. ${ }^{2}$ Dept of Pharmacology-Clinical Pharmacy, School of Pharmacy, Institut \\ Teknologi Bandung, Bandung, Indonesia. \\ Correspondence: Reinoud Gosens, A. Deusinglaan 1, 9713 AV Groningen, The Netherlands. \\ E-mail: r.gosensarug.nl
}

@ERSpublications

An epithelial-mesenchymal interaction governed by HSP60-regulated PRMT1 expression regulates airway remodelling and is reversed by bronchial thermoplasty http://bit.ly/2NDCKGW

Cite this article as: Rahmawati SF, Gosens R. Hot off the press: downregulation of PRMT1 for long-lasting effects of bronchial thermoplasty. Eur Respir J 2019; 54: 1901898 [https://doi.org/10.1183/13993003.018982019].

Asthma is broadly defined as a group of clinical symptoms with reversible expiratory airflow limitation or bronchial hyperresponsiveness with or without airway inflammation [1]. These symptoms are associated with airway remodelling, particularly in severe asthma patients, which is characterised by alterations of the tissue structure and cells within the airway, including increased submucosal extracellular matrix deposition, reticular basement membrane (RBM) thickening, airway smooth muscle (ASM) hyperplasia and hypertrophy, bronchial microvascular remodelling and mucous cell metaplasia [2-4].

Bronchial thermoplasty (BT) is an endoscopic procedure with a localised delivery of radiofrequency waves leading to heating $\left(65^{\circ} \mathrm{C}\right)$ of the airway tissue [5]. This procedure has been described as the first asthma treatment that targets airway remodelling directly instead of indirectly by modulating airway inflammation and airway hyperresponsiveness [6]. Long-term follow-up studies of three randomised controlled trials pointed out that BT reduces patients' asthma exacerbations, emergency department visits and hospitalisation up to 5 years after BT treatment [7-9]. This suggests that the improvement in lung function after BT might be a long-lasting effect. Indeed, BT decreased ASM mass and type I collagen deposition within the RBM [10]. In addition, BT lowered submucosal nerve density, ASM-associated nerve density and the number of epithelial neuroendocrine cells. These results were associated with several clinical outcomes, including the Asthma Control Test (ACT) scores, the number of exacerbations and emergency department visits at 3 and 12 months after BT [11]. The effect on the reduction in airway remodelling is likely not merely explained by a direct acute effect of thermal injury and must be due to an alternative biological mechanism triggered in response to BT [12]. Although these clinical and histopathological findings have significantly expanded our knowledge on BT, and revealed that its mode of action most likely goes well beyond the originally proposed selective impact on airway smooth muscle, we still lack a detailed mechanistic understanding of how BT regulates the remodelling process.

Epithelial-mesenchymal communication is a major mechanism that regulates airway remodelling. In this issue of the European Respiratory Journal, Sun et al. [13] hypothesised that crosstalk between epithelial cells and fibroblasts would underpin the beneficial effects of BT. They hypothesised that epithelial secreted 
factors would control epigenetic mechanisms in the fibroblast to drive long-lasting effects of BT. In their studies, they direct their attention primarily to protein arginine methyltransferase-1 (PRMT1). PRMT1 is the predominant PRMT enzyme in mammalian cells that methylates arginine residues on histones and other proteins. PRMT1 has been shown to have roles in the pathogenesis of lung cancer, pulmonary fibrosis, pulmonary hypertension, COPD and asthma [14]. Deletion of PRMT1 in mouse embryonic fibroblasts leads to DNA damage and reduced cell proliferation [15]. Therefore, it is of interest to study the role of PRMT1 in fibroblast proliferation leading up to airway remodelling in patients with severe asthma before and after undergoing BT.

In this study, the authors collected bronchoalveolar lavage fluid (BALF) from eight patients and primary bronchial epithelial cells (PBECs) from 62 patients with severe asthma before and after undergoing BT treatment. The PBECs were grown and collected. The supernatant from PBEC culture and BALF from patients was applied to primary fibroblasts. In these airway fibroblasts, they found increased PRMT1 expression, cell proliferation and mitochondria mass in response to BALF collected before BT, which were not observed in response to BALF collected after BT. In parallel, they observed downregulation of miR-19a as well as increased extracellular signal-regulated kinase-1/2 (ERK1/2) activity in response to BALF collected before BT, whereas BALF collected after BT did not show such changes. This is of importance as miR-19a is a repressor of ERK1/2 activity and PRMT1 expression [16]. CCAAT enhancer-binding protein- $\beta(\mathrm{C} / \mathrm{EBP} \beta)$ in turn is a negative regulator of miR-19a [17].

The authors further report that culture supernatant from airway epithelial cells collected after BT reduced C/EBP $\beta$ expression, ERK $1 / 2$ activity, peroxisome proliferator-activated receptor- $\gamma$ coactivator- $1 \alpha$ (PGC1 $\alpha$ ), heat shock protein-60 (HSP60), PRMT1 and mitochondria mass in airway fibroblasts compared to culture supernatant from epithelial cells obtained before BT. These results confirm that cellular crosstalk between epithelial cells and fibroblasts is key to some of the persistent changes observed after BT. Furthermore, downregulation of PRMT1 by siRNA reduced the expression of C/EBP $\beta$ and the proliferative effect of culture supernatant from epithelial cells before BT, indicating a crucial role of PRMT1 in this response. Collectively, these results show that both BALF and epithelial culture supernatant after BT decreased the proliferation of fibroblasts through a mechanism involving upregulation of miR-19a leading to downregulation of ERK1/2, PRMT1 and C/EBP $\beta$.

To elucidate the secreted factor that initiates this communication, the authors performed proteomic analysis on BALF and transcriptomic analysis on epithelial cells. They found that HSP60 expression was downregulated after BT. HSP60 is a chaperone protein expressed in mitochondria that is involved in the transportation and refolding of proteins from the cytoplasm into the mitochondrial matrix. In addition, HSP60 is a type of protein that is induced after subjecting cells to a stress response such as heat [18]. HSP60 released into the extracellular space has been described to function as a danger signal associated with autoimmune diseases, cancer, and inflammation as well as having direct interactions with molecules in various cell compartments [19]. Direct stimulation of lung fibroblasts with human recombinant HSP60 significantly increased the expression of $\mathrm{C} / \mathrm{EBP} \beta$, PRMT1 and PGC1 $\alpha$, confirming the crucial role of this intercellular signalling intermediate. Taken together, this indicates that there is an epithelial-mesenchymal interaction governed by HSP60-regulated PRMT1 expression that plays a role in airway remodelling and is reversed by BT (figure 1).

In this study, it was also found that PBECs obtained from asthma patients proliferated significantly faster after BT compared to those obtained before BT. This is in apparent contrast with previous study by PRETOLAni et al. [11] who did not observe any effect of BT on regenerating epithelium. This difference is likely explained by the difference in measurement methods and timing. Pretolani et al. [11] utilised histopathological evaluation of epithelium in situ, whereas Sun et al. [13] used a method for quantifying proliferation of epithelial cells ex vivo. In the clinical study in situ, the first follow-up measurement was taken after 3 months, which well exceeds the time window involved in cell proliferation of airway epithelial cells. Thus, it is not possible to infer from the Pretolani et al. [11] study whether there were changes in epithelial cell proliferation as captured in vitro by Sun et al. [13].

The study by Sun et al. [13] is the first one that shows the role of BT on PRMT1 expression, highlighting its role in airway remodelling. Further intriguing questions arise from this study and need to be explored. The first one is related to the mechanism of epithelial secreted HSP60 and the effects of BT hereon. It has been previously shown that stimulation by $\mathrm{H}_{2} \mathrm{O}_{2}$ to induce oxidative stress in cells increased HSP60 expression by epithelial cells into the extracellular medium through NFkB-p65 [20]. It would be of interest to study whether this is also the case with BT stimulation. It also interesting to study the epithelial secreted HSP60 signalling pathway as this appears to be a primary regulatory route towards PRMT1 expression in fibroblasts. For example, which receptors and signalling effectors are involved in fibroblast signalling in response to HSP60 and are these potential targets for drugs? 
Severe asthma before bronchial thermoplasty

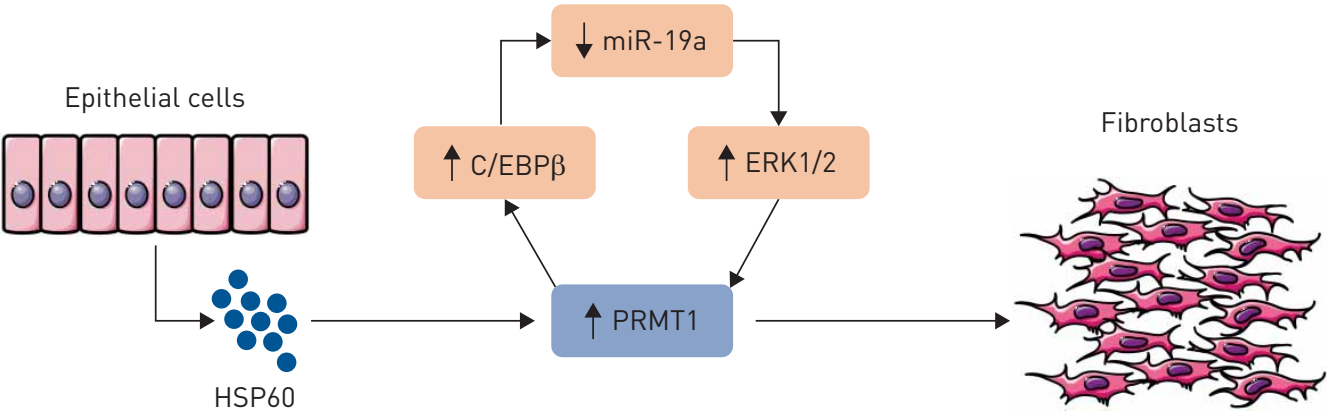

Severe asthma after bronchial thermoplasty
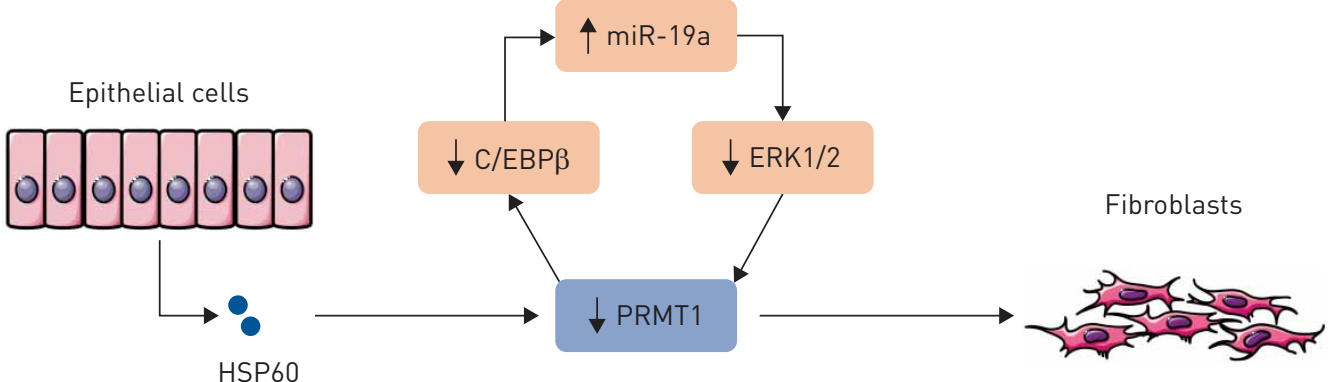

FIGURE 1 Schematic representation of the effect of bronchial thermoplasty on airway epithelial-mesenchymal communication. Bronchial thermoplasty reduces the release of heat shock protein-60 (HSP60) from epithelial cells, which in turn diminishes the expression of extracellular signal-regulated kinase-1/2 (ERK1/2), protein arginine methyltransferase-1 (PRMT1) and CCAAT enhancer-binding protein- $\beta$ (C/EBP $\beta$ ), whereas the expression of miR-19a is restored.

The second question is about uncovering the role of PRMT1 activity in regulating fibroblast responses. Other studies have shown that PRMT1 is responsible for modulating epithelial to mesenchymal transition through regulation of ZEB1 expression by histone modification of H4R3me2 [21] and through arginine 34 methylation of Twist1, resulting in E-cadherin repression [22]. In addition, PRMT1 methylates the epidermal growth factor receptor [23]. It would be of interest to also identify these possible pathways and the substrate(s) that are methylated by PRMT1 in fibroblasts, explaining its effects on airway remodelling.

Collectively, the study of Sun et al. [13] contributes to our understanding of the mechanisms involved in the beneficial effects of BT. They report sustained effects on epithelial-mesenchymal interactions in response to BT, characterised by a downregulated HSP60-PRMT1 signalling pathway. These new findings inspire not only further investigations into the pathways and epigenetic mechanisms regulated by BT, but also other potential therapeutic approaches on this signalling pathway, such as inhibition of HSP60 or PRMT1, that could potentially reduce the fibroblast responses associated with airway remodelling in asthma.

Conflict of interest: S.F. Rahmawati has nothing to disclose. R. Gosens reports grants from Boehringer Ingelheim, Chiesi Farmaceutici and Aquilo, outside the submitted work.

Support statement: This work was supported by the Lembaga Pengelola Dana Pendidikan/Indonesia Endowment Fund for Education (LPDP). Funding information for this article has been deposited with the Crossref Funder Registry.

\section{References}

Wenzel SE. Asthma phenotypes: the evolution from clinical to molecular approaches. Nat Med 2012; 18: 716-725. James AL, Wenzel S. Clinical relevance of airway remodelling in airway diseases. Eur Respir J 2007; 30: 134-155. Jeffery PK. Remodeling in asthma and chronic obstructive lung disease. Am J Respir Crit Care Med 2001; 164: 28. Pare PD, Roberts CR, Bai TR, et al. The functional consequences of airway remodeling in asthma. Monaldi Arch Chest Dis 1997; 52: 589-596.

5 Menzella F, Lusuardi M, Galeone C, et al. Bronchial thermoplasty and the role of airway smooth muscle: are we on the right direction? Ther Clin Risk Manag 2017; 13: 1213-1221. 
d'Hooghe JNS, Ten Hacken NHT, Weersink EJM, et al. Emerging understanding of the mechanism of action of bronchial thermoplasty in asthma. Pharmacol Ther 2018; 181: 101-107.

7 Thomson NC, Rubin AS, Niven RM, et al. Long-term (5 year) safety of bronchial thermoplasty: Asthma Intervention Research (AIR) trial. BMC Pulm Med 2011; 11: 8.

8 Wechsler ME, Laviolette M, Rubin AS, et al. Bronchial thermoplasty: long-term safety and effectiveness in patients with severe persistent asthma. J Allergy Clin Immunol 2013; 132: 1295-1302.

9 Pavord ID, Thomson NC, Niven RM, et al. Research in Severe Asthma Trial Study Group. Safety of bronchial thermoplasty in patients with severe refractory asthma. Ann Allergy Asthma Immunol 2013; 111: 402-407.

10 Chakir J, Haj-Salem I, Gras D, et al. Effects of bronchial thermoplasty on airway smooth muscle and collagen deposition in asthma. Ann Am Thorac Soc 2015; 12: 1612-1618.

11 Pretolani M, Bergqvist A, Thabut G, et al. Effectiveness of bronchial thermoplasty in patients with severe refractory asthma: clinical and histopathologic correlations. J Allergy Clin Immunol 2017; 139: 1176-1185.

12 Chernyavsky IL, Russell RJ, Saunders RM, et al. In vitro, in silico and in vivo study challenges the impact of bronchial thermoplasty on acute airway smooth muscle mass loss. Eur Respir J 2018; 51: 1701680.

13 Sun Q, Fang L, Roth M, et al. Bronchial thermoplasty decreases airway remodelling by blocking epithelium-derived heat shock protein-60 secretion and protein arginine methyltransferase-1 in fibroblasts. Eur Respir J 2019; 54: 1900300.

14 Wei H, Mundade R, Lange KC, et al. Protein arginine methylation of non-histone proteins and its role in diseases. Cell Cycle 2014; 13: 32-41.

15 Yu Z, Chen T, Hebert J, et al. A mouse PRMT1 null allele defines an essential role for arginine methylation in genome maintenance and cell proliferation. Mol Cell Biol 2009; 29: 2982-2996.

16 Sun Q, Liu L, Wang $\mathrm{H}$, et al. Constitutive high expression of protein arginine methyltransferase 1 in asthmatic airway smooth muscle cells is caused by reduced microRNA-19a expression and leads to enhanced remodeling. J Allergy Clin Immunol 2017; 140: 51-524. e3.

17 Yan Y, Hanse EA, Stedman K, et al. Transcription factor C/EBP-beta induces tumor-suppressor phosphatase PHLPP2 through repression of the miR-17-92 cluster in differentiating AML cells. Cell Death Differ 2016; 23: 1232-1242.

18 Schlesinger MJ. Heat shock proteins. J Biol Chem 1990; 265: 12111-12114.

19 Cappello F, Marino Gammazza A, Palumbo Piccionello A, et al. Hsp60 chaperonopathies and chaperonotherapy: targets and agents. Expert Opin Ther Targets 2014; 18: 185-208.

20 Cappello F, Caramori G, Campanella C, et al. Convergent sets of data from in vivo and in vitro methods point to an active role of Hsp60 in chronic obstructive pulmonary disease pathogenesis. PLoS One 2011; 6: e28200.

21 Gao Y, Zhao Y, Zhang J, et al. The dual function of PRMT1 in modulating epithelial-mesenchymal transition and cellular senescence in breast cancer cells through regulation of ZEB1. Sci Rep 2016; 6: 19874.

22 Avasarala S, Van Scoyk M, Karuppusamy Rathinam MK, et al. PRMT1 is a novel regulator of epithelial-mesenchymal-transition in non-small cell lung cancer. J Biol Chem 2015; 290: 13479-13489.

23 Liao HW, Hsu JM, Xia W, et al. PRMT1-mediated methylation of the EGF receptor regulates signaling and cetuximab response. J Clin Invest 2015; 125: 4529-4543. 